\title{
The OB associations LH 101 and LH 104 in the HII region N158 of the $\mathrm{LMC}^{\star}$
}

\author{
G. Testor ${ }^{1}$ and V. Niemela ${ }^{2, \star \star, \star \star \star}$ \\ 1 Observatoire de Paris, Section de Meudon, F-92195 Meudon Cedex, DAEC, France \\ 2 Facultad de Ciencias Astronómicas y Geofísicas, Universidad Nacional de La Plata, 1900 La Plata, Argentina
}

Received October 30, 1997; accepted January 28, 1998

\begin{abstract}
We present photometric and spectroscopic observations of stars in the Large Magellanic Cloud OB associations LH 101 and LH 104, located in the HII region N158, which we have also imaged. From our observations we have constructed upper $\mathrm{H}-\mathrm{R}$ diagrams for these $\mathrm{OB}$ associations, which we find to consist mainly of three populations, one of $2-6 \mathrm{Myr}$ for the stars inside the northern bubble (LH 104), and two populations in the southern HII region (LH 101), one of $\leq 2 \mathrm{Myr}$ and the other one aged 3-6 Myr. We have obtained for LH 101 a normal IMF, with a slope of $\Gamma=-1.29 \pm 0.20$ whereas for LH 104 the IMF is flatter with a slope of $\Gamma=-1.05 \pm 0.12$. These IMF slopes are consistent with that of other OB associations in the LMC. Our observations reveal in the region of LH 101 the presence of both unevolved and evolved very massive stars, whose ionizing flux is in excess of that derived from our $\mathrm{H} \beta$ images of the HII region. The northwest nebulosity in the region of LH 101 thus appears to be matter bound.
\end{abstract}

Key words: Magellanic Clouds - open clusters and associations: individual: LMC-LH 101, LH 104 - stars: early type - stars: Wolf-Rayet

\section{Introduction}

The Large Magellanic Cloud (LMC) is rich in HII regions and young $\mathrm{OB}$ associations and, because of its known and relatively small distance, it is well suited to CCD observations and is an ideal laboratory in which to study the

Send offprint requests to: G. Testor (G.Testor@obspm.fr)

* Based on observations obtained at ESO, La Silla, Chile.

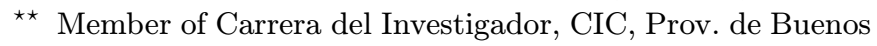
Aires, Argentina.

$\star \star \star$ Visiting Astronomer, CASLEO, operated under agreement between CONICET, SeCyT, and the Universities of La Plata, Córdoba and San Juan, Argentina.

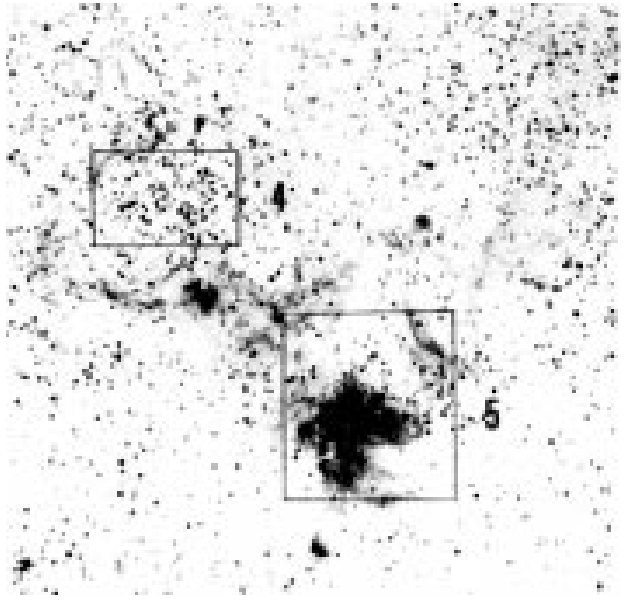

Fig. 1. Reproduction from the ESO B sky survey of the N158 complex. The observed aeras are outlined and numbered. North is up and east to the left

formation and evolution of massive stars. We therefore embarked on detailed studies of such regions. In this paper we will deal with the OB associations LH 101 and LH 104 (Lucke \& Hodge 1970), which were also included in the photographic photometry of $\mathrm{OB}$ associations in the Magellanic Clouds performed by Westerlund (1961). These associations are inside the isolated elongated giant HII region N 158 (Henize 1956). This HII region is located $20^{\prime}$ south of the nucleus of 30 Dor and stretches over $11^{\prime}$ (equivalent to $\sim 160 \mathrm{pc}$ ) in the NE-SW direction. Its northern part is composed of a superbubble around the OB association LH 104, while the southern part containing the OB association LH 101 is a diffuse HII region which is characterized by three bright zones. The whole region belongs to a large complex of ionized gas in the northern part of a giant molecular cloud bordering the two young neighbour associations in the nebulae N159 and N160 (Israel et al. 1996). 


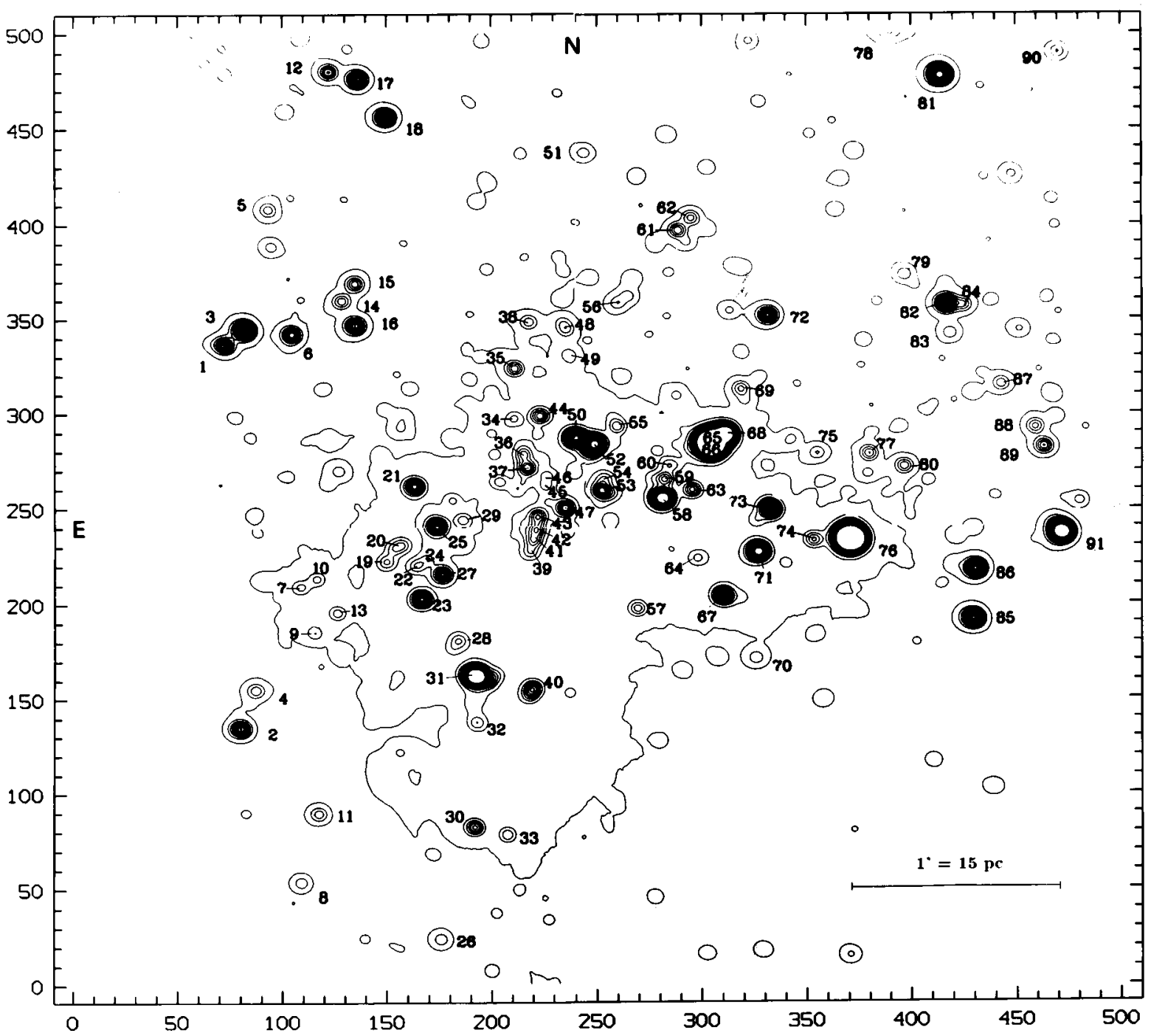

Fig. 2. Identification chart for the stars of the Zone 5 in LH 101. The isophotal map was obtained from the exposure $B$ image

Lortet \& Testor (1988) have studied the morphology of this region, and described conspicuous sequential star formation from the stellar association LH 104 toward the most excited parts of N158C. The sequential star formation in N158 is strengthened by recent studies showing that LH 104 is dominated by B stars (Schild \& Testor 1992) and LH 101 by unevolved and evolved O-type massive stars (Testor \& Niemela 1996), suggesting that this region is an active site of very massive star formation.

Integrated $\mathrm{H} \alpha$ and $\mathrm{H} \beta$ fluxes of $\mathrm{N} 158$ through diaphragms have been measured by Kennicut \& Hodge (1986) and Caplan \& Deharveng (1985). Jones et al. (1986) detected two infrared sources in LH 101: NIR 1 and NIR 2. NIR 1 has colours typical of a reddened $\mathrm{O}$ star of luminosity $\sim 210^{6} L_{\odot}$

The region of $\mathrm{N} 158$ is also the third richest area of the LMC for WR stars. Nevertheless, a detailed examination of the stellar content of LH 101/104 is lacking, perhaps because the bright nebulosity hinders accurate stellar photometry. In this paper we present:

- in Sect. 2 the data base, consisting of new observational material of stars in LH 101, namely $U B V$ CCD photometry and spectra, and previous imagery and data. The data reduction closely follows the study of LH 90 by Testor \& Schild (1993).

- in Sect. 3 a description of the spectra of peculiar stars, and the $\mathrm{H}-\mathrm{R}$ diagrams of the two associations with a description of their age structure.

- in Sects. 4 and 5 the determination of the initial mass function (IMF) for both OB associations, and for the HII region of LH 101 a comparison between its $\mathrm{H} \alpha$ luminosity and the Lyman ionizing flux. 


\section{Observations and reductions}

\subsection{UBV photometry}

The photometry of LH 101 was carried out during an observing run from December 28, 1992, to January 1, 1993, at the European Southern Observatory (ESO), La Silla, Chile, with the 3.6-m telescope and EFOSC, the ESO Faint Object Spectrograph and Camera (Melnick et al. 1989). The detector was a Tektronix CCD with $512 \times 512$ pixels, providing an image scale of 0.63 " / pixel. The seeing during the run was about $1.3^{\prime \prime}$ and the images of LH 101 through standard $U, B$ and $V$ filters were obtained under slightly non-photometric conditions. The EFOSC field of view was $3.5^{\prime} \times 5.6^{\prime}$. The values of the instrumental $u b v$ magnitudes were obtained with the DAOPHOT package (Stetson 1987) based on a PSF determination, as implemented in the MIDAS system. DAOPHOT is especially well adapted to crowded fields, as those in the Magellanic Clouds. A color correction was applied using the stars observed in the standard field PG $0918+028$ of Landolt (1992). The following relations between the Johnson $U B V$ magnitude and the instrumental magnitude $u b v$ were obtained:

$V=v+(b-v) \times 0.08+$ cte

$B-V=(b-v) \times 1.16+$ cte

$U-B=(u-b) \times 1.00+$ cte.

Because of variations of tranparency during the observations the constant offset was derived from some isolated stars of our association photometered by Lucke (1972).

The typical errors calculated by DAOPHOT for the $V, B-V$ and $U-B$ of objects brighter than 15 th mag are less than $0.01,0.015$ and $0.02 \mathrm{mag}$, respectively. In the range of $15-16$ mags they are less than $0.015,0.03$ and 0.03 mag. Table 1 lists new $U B V$ photometry for stars brighter than $V \sim 17$. The region in LH 101 for which both $U B V$ magnitudes and spectral types were obtained is outlined in Fig. 1. We will refer to this area as zone 5, following the numbering of Schild \& Testor (1992) (Paper I). An enlarged finder chart is shown in Fig. 2 .

Concerning LH 104, except when noted otherwise, the photometric observations used as data base are from Paper I.

\subsection{Spectroscopic observations}

Spectra of stars in LH 101 were observed during the same observing run as the photometry, also using EFOSC with the $3.6-\mathrm{m}$ telescope. The spectra were obtained both in single-slit and MOS mode (multi-object spectroscopy) with a grism providing a reciprocal dispersion of $120 \AA / \mathrm{mm}$ and yielding a resolution of $\sim 8 \AA$ (3 pixels at $2.7 \AA /$ pixel). The detector was the same CCD as used in photometry.

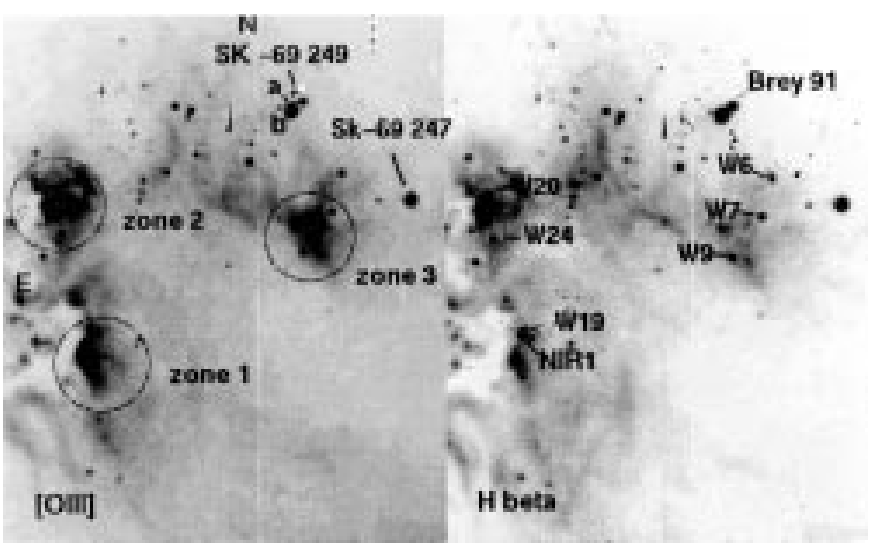

Fig. 3. CCD frames of LH101 in N158C through [OIII] and $\mathrm{H} \beta$ filter. A few stars are identified with their Westerlund, Breysacher or Sanduleak identification. The star W19 is superimposed with the I.R source $\mathrm{N}^{\circ} 1$ in Jones et al. (1986) and the excited HII zones are indicated

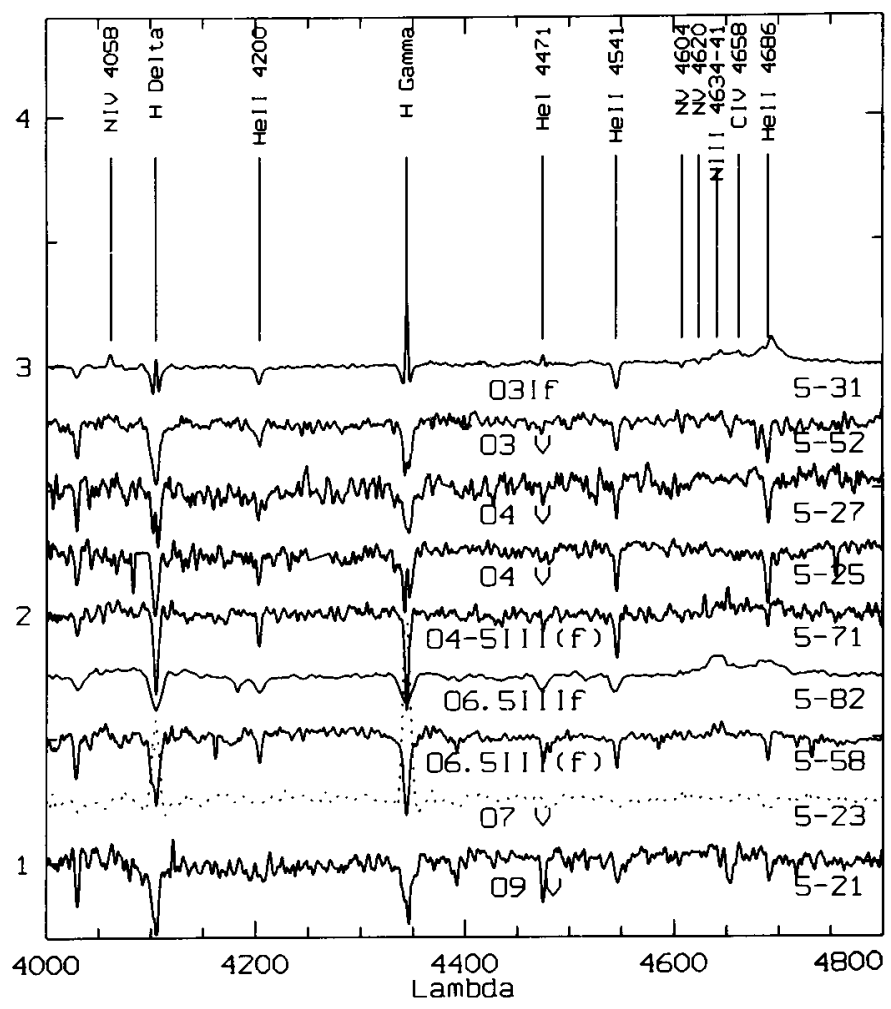

Fig. 4. Selected rectified spectra of evolved and unevolved stars in LH 101

Some additional long slit spectra were obtained with the ESO $1.5 \mathrm{~m}$ telescope during two observing runs in January and December 1995, using the B\&C spectrograph and the holographic grating, giving a reciprocal dispersion of $30 \AA / \mathrm{mm}$. In the range $\lambda \lambda 4000-5200 \AA$ this grating allowed to obtain a spectral resolution $\delta \lambda \sim 1 \AA$ corresponding to $\sim 2$ pixels. The detector was a $2000 \times 2000$ pixels Ford high resolution CCD. The pixel size of $15 \mu$ projected to $0.7 \times 0.7^{\prime \prime}$ on the sky. 
Table 1. Observational data and deduced values of $E(B-V), M_{\mathrm{bol}}$ and $T_{\text {eff }}$ for the stars of the LH 101 association

\begin{tabular}{|c|c|c|c|c|c|c|c|c|c|c|c|}
\hline Id & Other & $\alpha(2000)$ & $\delta(2000)$ & $V$ & $B-V$ & $U-B$ & $Q$ & $E(B-V)$ & $M_{\mathrm{bol}}$ & $\log \left(T_{\text {eff }}\right)$ & Type \\
\hline $5-1$ & $26^{w}$ & 53926.60 & -692847.7 & 14.48 & -0.11 & -0.88 & -0.85 & 0.18 & -7.42 & 4.45 & \\
\hline $5-2$ & & 53925.72 & -693054.9 & 14.43 & -0.17 & -1.00 & -0.81 & 0.14 & -7.76 & 4.51 & \\
\hline $5-3$ & $25^{w}$ & 53925.48 & -692842.8 & 13.59 & -0.02 & -0.86 & -0.88 & 0.18 & -7.34 & 4.32 & B1 Ib \\
\hline $5-4$ & & 53924.83 & -693042.2 & 15.60 & -0.13 & -0.93 & -0.85 & 0.16 & -6.40 & 4.47 & \\
\hline $5-5$ & & 53924.07 & -69282.7 & 15.66 & -0.11 & -0.84 & -0.76 & 0.16 & -5.97 & 4.41 & \\
\hline $5-6$ & & 53922.72 & -692844.4 & 14.56 & -0.14 & -0.99 & -0.84 & 0.18 & -8.20 & 4.59 & $\mathrm{O} 7-8 \mathrm{~V}$ \\
\hline $5-7$ & & 53922.27 & -69308.0 & 15.82 & -0.06 & -0.86 & -0.82 & 0.23 & -6.31 & 4.46 & \\
\hline $5-8$ & & 53922.23 & -693146 . & 15.28 & 0.63 & 0.48 & 0.03 & * & * & $*$ & $f ?$ \\
\hline $5-9$ & & 53921.45 & -693023.1 & 15.79 & 0.12 & -0.71 & -0.79 & 0.40 & -6.74 & 4.44 & \\
\hline $5-10$ & & 53921.30 & -69305.4 & 15.81 & -0.06 & -0.88 & -0.84 & 0.24 & -6.47 & 4.48 & \\
\hline $5-11$ & & 53921.22 & -693123.4 & 15.04 & 0.48 & 0.06 & -0.29 & * & $*$ & * & $f ?$ \\
\hline $5-12$ & & 53920.58 & -692717.4 & 15.07 & -0.21 & -1.05 & -0.90 & 0.11 & -7.10 & 4.53 & \\
\hline $5-13$ & & 53920.12 & -693016.6 & 15.49 & 0.15 & -0.74 & -0.85 & 0.45 & -7.46 & 4.48 & \\
\hline $5-14$ & & 53919.85 & -692833.3 & 15.26 & -0.06 & -0.86 & -0.82 & 0.23 & -6.87 & 4.46 & \\
\hline $5-15$ & & 53919.08 & $-6928 \quad 27.5$ & 15.00 & -0.05 & -0.80 & -0.76 & 0.22 & -6.83 & 4.41 & \\
\hline $5-16$ & & 53919.07 & -692841.5 & 14.35 & -0.08 & -0.80 & -0.74 & 0.21 & -7.69 & 4.46 & B0 III \\
\hline $5-17$ & & 53918.92 & -692719.9 & 13.65 & 0.50 & 0.14 & -0.20 & * & $*$ & * & $\mathrm{G}^{f}$ \\
\hline $5-18$ & & 53917.32 & -692732.5 & 14.29 & -0.15 & -0.97 & -0.86 & 0.08 & -7.15 & 4.50 & B0 I \\
\hline $5-19$ & & 53917.28 & -692959.7 & 15.45 & -0.02 & -0.92 & -0.91 & 0.30 & -7.38 & 4.54 & \\
\hline $5-20$ & & 53916.46 & -692954.3 & 15.68 & -0.19 & -0.93 & -0.79 & 0.09 & -5.88 & 4.44 & \\
\hline $5-21$ & $22^{w}$ & 53915.69 & -692935.0 & 14.31 & -0.06 & -0.93 & -0.88 & 0.25 & -8.34 & 4.54 & O9 V \\
\hline $5-22$ & & 53915.58 & -69300.9 & 15.83 & -0.05 & -0.75 & -0.71 & 0.20 & -5.71 & 4.37 & \\
\hline $5-23$ & $21^{w}$ & 53915.29 & -693011.9 & 14.15 & -0.09 & -0.87 & -0.81 & 0.23 & -8.56 & 4.59 & $\mathrm{O} 7 \mathrm{~V}$ \\
\hline $5-24$ & & 53914.99 & -69 2959.1 & 16.29 & 0.01 & -0.80 & -0.81 & 0.30 & -5.99 & 4.45 & \\
\hline $5-25$ & $20^{w}$ & 53914.41 & -692948.1 & 14.20 & -0.04 & -0.98 & -0.95 & 0.29 & -9.41 & 4.67 & $\mathrm{O} 4 \mathrm{~V}$ \\
\hline $5-26$ & & 53914.23 & -69324.5 & 16.00 & -0.07 & -0.84 & -0.79 & 0.21 & -5.92 & 4.43 & \\
\hline $5-27$ & $24^{w}$ & 53914.10 & -69303.8 & 14.58 & -0.10 & -1.00 & -0.93 & 0.23 & -8.93 & 4.69 & $\mathrm{O} 4 \mathrm{~V}$ \\
\hline $5-28$ & & 53913.18 & -693025.7 & 15.61 & -0.05 & -0.94 & -0.90 & 0.27 & -7.07 & 4.53 & \\
\hline $5-29$ & & 53912.94 & -692946.3 & 15.69 & 0.02 & -0.70 & -0.71 & 0.27 & -6.07 & 4.37 & \\
\hline $5-30$ & & 53912.29 & -693127.7 & 15.02 & -0.17 & -0.94 & -0.82 & 0.12 & -6.77 & 4.46 & \\
\hline $5-31$ & $19^{w}$ & 53912.20 & -693037.6 & 12.50 & -0.12 & -0.90 & -0.81 & 0.19 & -10.6 & 4.65 & O3 If \\
\hline $5-32$ & & 53912.14 & -693053.1 & 15.64 & -0.03 & -0.89 & -0.87 & 0.28 & -6.91 & 4.50 & \\
\hline $5-33$ & & 53910.39 & -693130.2 & 15.75 & -0.24 & -1.00 & -0.82 & 0.05 & -5.84 & 4.46 & \\
\hline $5-34$ & & 5399.95 & -692912.5 & 16.05 & -0.12 & -0.83 & -0.74 & 0.14 & -5.44 & 4.39 & \\
\hline $5-35$ & $19^{l}$ & 5399.92 & -692856.1 & 15.01 & -0.01 & -0.75 & -0.7 & 0.26 & -6.87 & 4.40 & \\
\hline $5-36$ & & 5399.41 & -692924.4 & 15.32 & 0.06 & -0.75 & -0.79 & 0.35 & -7.07 & 4.45 & B0 III \\
\hline $5-37$ & & 5399.19 & -692928.9 & 14.71 & -0.07 & -0.71 & -0.66 & 0.17 & -6.48 & 4.33 & \\
\hline $5-38$ & & 5399.11 & -692840.5 & 15.66 & -0.03 & -1.05 & -1.03 & 0.33 & -7.93 & 4.65 & \\
\hline $5-39$ & & 5399.03 & -692956.5 & 15.63 & -0.03 & -0.77 & -0.75 & 0.24 & -6.19 & 4.40 & \\
\hline $5-40$ & $17^{w}$ & 5398.93 & -693041.7 & 14.75 & -0.10 & -1.00 & -0.92 & 0.22 & -7.89 & 4.55 & \\
\hline $5-41$ & & 5398.85 & -692952.7 & 15.21 & -0.15 & -0.90 & -0.79 & 0.13 & -6.44 & 4.43 & \\
\hline $5-42$ & & 5398.66 & -69 2949.2 & 14.95 & -0.03 & -0.87 & -0.85 & 0.27 & -7.45 & 4.48 & \\
\hline $5-43$ & & 5398.59 & -692945.1 & 14.85 & 0.08 & -0.70 & -0.76 & 0.35 & -7.34 & 4.41 & \\
\hline $5-44$ & & 5398.48 & -69 2911.8 & 14.94 & -0.10 & -0.75 & -0.68 & 0.21 & -7.27 & 4.52 & O9 III \\
\hline $5-45$ & & 5398.14 & -692934.9 & 16.20 & 0.08 & -0.80 & -0.86 & 0.38 & -6.60 & 4.49 & \\
\hline $5-46$ & & 5398.06 & -692931.5 & 16.65 & -0.20 & -0.85 & -0.71 & 0.05 & -4.38 & 4.36 & \\
\hline $5-47$ & & 5397.01 & -692942.3 & 14.59 & -0.06 & -0.78 & -0.73 & 0.25 & -7.98 & 4.53 & O9 IV \\
\hline $5-48$ & $18^{l}$ & 5396.98 & -692842.9 & 15.70 & -0.13 & -0.94 & -0.85 & 0.17 & -6.38 & 4.48 & \\
\hline $5-49$ & & 5396.80 & -692851.6 & 16.08 & -0.10 & -0.87 & -0.80 & 0.18 & -5.81 & 4.44 & \\
\hline $5-50$ & $15^{w}$ & 5396.43 & -692919.1 & 13.57 & 0.05 & -0.82 & -0.86 & 0.32 & -8.64 & 4.42 & B0.5 III \\
\hline $5-51$ & & 5396.03 & -692744.1 & 15.35 & 0.59 & 0.47 & 0.04 & 0.58 & $*$ & $*$ & $f ?$ \\
\hline $5-52$ & $14^{w}$ & 5395.41 & -69 2920.7 & 13.41 & -0.15 & -0.89 & -0.78 & 0.18 & -10.00 & 4.68 & $\mathrm{O} 3 \mathrm{~V}$ \\
\hline $5-53$ & $16^{w}$ & 5395.03 & -692936.2 & 14.60 & -0.13 & -0.74 & -0.64 & 0.10 & -6.31 & 4.32 & \\
\hline $5-54$ & & 5394.90 & -692932.3 & 16.32 & -0.16 & -0.75 & -0.63 & 0.07 & -4.44 & 4.31 & \\
\hline $5-55$ & & 5394.14 & -692914.9 & 15.33 & -0.04 & -0.70 & -0.67 & 0.24 & -6.82 & 4.45 & B0.5 V \\
\hline $5-56$ & & 5394.12 & -692834.0 & 16.06 & -0.14 & -0.79 & -0.70 & 0.11 & -5.11 & 4.36 & \\
\hline
\end{tabular}


Table 1. continued

\begin{tabular}{|c|c|c|c|c|c|c|c|c|c|c|c|}
\hline Id & Other & $\alpha(2000)$ & $\delta(2000)$ & $V$ & $B-V$ & $U-B$ & $Q$ & $E(B-V)$ & $M_{\mathrm{bol}}$ & $\log \left(T_{\text {eff }}\right)$ & Type \\
\hline $5-57$ & & 5392.99 & -693015.5 & 15.41 & -0.09 & -0.80 & -0.73 & 0.17 & -6.10 & 4.38 & \\
\hline $5-58$ & $12^{w}$ & 5391.54 & -692938.9 & 13.06 & -0.11 & -0.89 & -0.81 & 0.21 & -9.75 & 4.59 & O6.5III(f) \\
\hline $5-59$ & & 5391.42 & -692932.4 & 15.15 & -0.10 & -0.75 & -0.68 & 0.14 & -6.03 & 4.34 & \\
\hline $5-60$ & & 5391.18 & -692927.9 & 15.89 & -0.1 & -0.70 & -0.63 & 0.13 & -5.02 & 4.31 & \\
\hline $5-61$ & & 5390.68 & -69289.9 & 15.02 & -0.09 & -0.77 & -0.71 & 0.22 & -7.45 & 4.53 & O9 V \\
\hline $5-62$ & & 53859.94 & -69285.7 & 15.36 & -0.09 & -0.81 & -0.75 & 0.17 & -6.22 & 4.40 & \\
\hline $5-63$ & $11^{l}$ & 53859.80 & -692935.9 & 15.02 & 0.01 & -0.98 & -0.99 & 0.32 & -7.48 & 4.48 & O9.5 V \\
\hline $5-64$ & & 53859.57 & -692959.0 & 15.97 & -0.12 & & -0.66 & 11 & & 4.33 & \\
\hline $5-65$ & Sk-69 249 & 53858.87 & -692921.6 & 11.44 & 0.02 & -0.85 & -0.99 & 29 & -11.5 & 4.56 & O7 If $^{b}$ \\
\hline $5-66$ & Sk-69 249 & 53858.87 & -692923.2 & 11.80 & -0.07 & -0.83 & -0.93 & 0.16 & -9.69 & 4.41 & B0 I \\
\hline $5-67$ & $9^{w}$ & 53858.09 & -693011.4 & 14.02 & -0.08 & -0.88 & -0.83 & 0.22 & -8.16 & 4.48 & O9.5 III \\
\hline $5-68$ & Brey 91 & 53858.04 & -692918.5 & 12.63 & -0.13 & -0.97 & -0.88 & 0.19 & -9.42 & 4.51 & WN9 \\
\hline $5-69$ & & 53857.09 & -69292.8 & 15.46 & -0.12 & -0.72 & -0.63 & 0.19 & -6.86 & 4.52 & $09.5 \mathrm{~V}$ \\
\hline $5-70$ & & 5385 & -693031.9 & 16.06 & -0.12 & -0.82 & -0.74 & 0.14 & -5.39 & 4.39 & \\
\hline $5-71$ & $7^{w}$ & 53856.08 & -692956.9 & 13.38 & -0.14 & -0.91 & -0.81 & 0.18 & -9.73 & 4.65 & O4-5 III \\
\hline $5-72$ & $16^{l}$ & 53855.53 & -692838.3 & 13.56 & 0.67 & 0.57 & 0.13 & * & $*$ & * & $\mathrm{G}^{f}$ \\
\hline $5-73$ & $6^{w}$ & 53855.40 & -692943.1 & 13.90 & -0.13 & -0.84 & -0.75 & 0.18 & -8.21 & 4.52 & O9 III \\
\hline $5-74$ & & 53852.92 & -692953.0 & 15.22 & -0.09 & -0.83 & -0.76 & & & & \\
\hline $5-75$ & & 53852.73 & -692924.1 & 15.93 & -0.10 & -0.72 & -0.65 & 0.14 & -5.12 & 4.32 & \\
\hline $5-76$ & Sk-69 247 & 53850.80 & -692953.7 & 10.42 & 0.17 & -0.69 & -0.81 & 0.46 & -8.95 & 4.05 & A0 Iae \\
\hline $5-77$ & & 53849.76 & -69 2924.3 & 15.24 & -0.09 & -0.77 & -0.70 & 0.18 & -6.75 & 4.44 & B0.7 V \\
\hline $5-78$ & $21^{l}$ & 53848.49 & -69270.7 & $10.17^{l}$ & $1.69^{l}$ & 2.30 & $*$ & * & $*$ & * & $\mathrm{G}^{f}$ \\
\hline $5-79$ & & 53847. & -692824.8 & 15.94 & -0 . & -0.6 & -0.62 & 0.17 & -6.00 & 45 & B1 V \\
\hline $5-80$ & $12^{l}$ & 53847.73 & -692928.5 & 15.26 & -0.10 & -0.78 & -0.71 & 0.16 & -6.78 & 4.52 & O9 III \\
\hline $5-81$ & & 53845.67 & -692718.9 & 13.22 & -0.02 & -0.68 & -0.63 & 0.22 & -7.94 & 4.30 & \\
\hline $5-82$ & $27^{w}$ & 53845.32 & -692834.7 & 13.81 & -0.08 & -0.77 & -0.71 & 0.24 & -9.13 & 4.59 & O6.5 III \\
\hline $5-83$ & & 53845.12 & -692844.4 & 15.99 & -0.12 & -0.66 & -0.58 & & -4.63 & & \\
\hline $5-84$ & & 53844.37 & -692835.0 & 15.37 & -0.03 & -0.62 & -0.60 & 0.19 & -5.59 & 4.28 & \\
\hline $5-85$ & $8^{l}$ & 53843.80 & -693019.3 & 13.69 & -0.01 & -0.72 & -0.71 & 0.29 & -9.11 & 4.55 & $\mathrm{O} 7.5 \mathrm{I}(\mathrm{f})$ \\
\hline $5-86$ & $9^{l}$ & 53843.68 & -69302.8 & 13.76 & -0.01 & -0.62 & -0.61 & 0.27 & -8.21 & 4.42 & B0.5 III \\
\hline $5-87$ & $14^{l}$ & 53842.18 & $\begin{array}{lll}-69 & 29 & 1.4\end{array}$ & 15.51 & -0.02 & -0.55 & -0.53 & 0.17 & -5.14 & 4.24 & \\
\hline $5-88$ & $13^{l}$ & 53840.24 & -692915.6 & 15.34 & -0.02 & -0.57 & -0.56 & 0.18 & -5.44 & 4.26 & \\
\hline $5-89$ & $12^{l}$ & 53839.73 & -692922.2 & 14.94 & -0.05 & -0.56 & -0.52 & 0.14 & -5.56 & 4.23 & \\
\hline $5-90$ & & 53838.95 & -692711.4 & 15.82 & -0.07 & -0.65 & -0.60 & 0.15 & -5.03 & 4.28 & \\
\hline $5-91$ & $10^{l}, 1^{w}$ & 53838.70 & -692950.9 & 12.07 & 0.44 & 0.15 & -0.10 & -0.14 & $*$ & * & G0: ${ }^{f}$ \\
\hline
\end{tabular}

Notes to Table 1:

Column 2: "Sk" refer to Sanduleak (1969), "Brey" to Breysacher (1981), ${ }^{l}$ to Lucke (1972) and ${ }^{w}$ to Westerlund (1961).

Column 5: ${ }^{l}$ Lucke's values.

Column 12: ${ }^{f}$ foreground star determined from spectra, ${ }^{f ?}$ possible foreground star supported by the observed color and ${ }^{b}$ spectroscopic binary.

All the spectroscopic raw data obtained at ESO were reduced with the MIDAS software package available at Paris-Meudon Observatory. The usual corrections like flatfielding and sky substraction were applied in a standard way. The spectra were wavelength calibrated with a helium-argon arc and then rectified.

In addition, spectra of a few stars in LH 104 and LH 101 were observed with the $2.15-\mathrm{m}$ telescope at Complejo Astronómico El Leoncito (CASLEO), San Juan, Argentina, during December 1996. These spectra were obtained with a $\mathrm{B} \& \mathrm{C}$ spectrograph with a grating giving a reciprocal dispersion of $120 \AA / \mathrm{mm}$. The detector was a PM512A UV coated $512 \times 512$ pixels CCD, with pixel size of $19 \mu$. The observed wavelength range was $\lambda \lambda 3800-$ $4900 \AA$. $\mathrm{A} \sim 2^{\prime}$ long slit was used, which eventually permitted the observation of several stars at the same time.

All the spectra obtained at CASLEO were reduced using IRAF package at La Plata Observatory.

\subsection{Monochromatic imaging}

Narrowband $\mathrm{H} \beta$, [OIII $] \lambda 5007$ and $[\mathrm{OII}] \lambda 3727$ images of the LH 101/104 complex were obtained with the Danish 1.5-m telescope at La Silla, Chile in 1985, as described by Lortet \& Testor (1988). The [OII] image of LH 104 
Table 2. Observational data and deduced values of $E(B-V), M_{\mathrm{bol}}$ and $T_{\text {eff }}$ for the stars of the LH 104 association

\begin{tabular}{|c|c|c|c|c|c|c|c|c|c|c|c|}
\hline Id & Other & $\alpha(2000)$ & $\delta(2000)$ & $V^{c}$ & $B-V^{c}$ & $U-B^{c}$ & $Q$ & $E(B-V)$ & $M_{\mathrm{bol}}$ & $\log \left(T_{\text {eff }}\right)$ & Type \\
\hline $4-1$ & & 53942.19 & -692429.01 & 15.57 & -0.08 & -0.88 & -0.82 & 0.16 & -5.45 & 4.23 & B2 III \\
\hline $4-2$ & & 53942.47 & -692259.00 & 16.09 & -0.06 & -0.88 & -0.84 & 0.20 & -5.73 & 4.41 & B1 V \\
\hline $4-3$ & & 53942.77 & -692352.01 & 16.16 & -0.09 & -0.95 & -0.89 & 0.15 & -5.16 & 4.34 & $\mathrm{~B} 2 \mathrm{~V}$ \\
\hline $4-4$ & & 53942.77 & -692345.99 & 14.76 & 0.06 & -1.05 & -1.09 & 0.32 & -7.43 & 4.41 & $\mathrm{~B} 1 \mathrm{~V}$ \\
\hline $4-5$ & & 53944.10 & -692353.99 & 16.37 & -0.01 & -0.87 & -0.86 & 0.23 & -5.19 & 4.34 & $\mathrm{~B} 2 \mathrm{~V}$ \\
\hline $4-6$ & & 53944.29 & -692336.00 & 15.66 & -0.04 & -0.87 & -0.84 & 0.20 & -5.81 & 4.34 & $\mathrm{~B} 2 \mathrm{~V}$ \\
\hline $4-7$ & & 53945.22 & -692449.99 & 16.74 & 0.00 & -0.82 & -0.82 & 0.24 & -4.85 & 4.34 & $\mathrm{~B} 2 \mathrm{~V}$ \\
\hline $4-8$ & & 53945.44 & -692321.00 & 17.40 & -0.02 & -0.84 & -0.83 & 0.27 & -4.89 & 4.46 & B1: \\
\hline $4-71$ & & 53945.58 & -692518.01 & 15.10 & -0.13 & -1.03 & -0.94 & 0.13 & -6.50 & 4.41 & B1 V \\
\hline $4-9$ & & 53947.13 & -69248.98 & 16.27 & -0.07 & -0.95 & -0.90 & 0.19 & -5.52 & 4.41 & B1 V \\
\hline $4-10$ & & 53947.52 & -692316.99 & 16.71 & 0.08 & -0.82 & -0.88 & 0.39 & -6.22 & 4.51 & B2: \\
\hline $4-11$ & & 53948.26 & -692414.01 & 15.60 & 0.01 & -0.86 & -0.87 & 0.27 & -6.16 & 4.38 & B1 III \\
\hline $4-12$ & & 53948.27 & -692340.99 & 15.79 & -0.15 & -0.93 & -0.82 & 0.14 & -6.08 & 4.46 & - \\
\hline $4-13$ & & 53948.66 & -692314.00 & 14.91 & -0.05 & -0.87 & -0.83 & 0.14 & -5.89 & 4.32 & B1 I \\
\hline $4-14$ & & 53949.03 & -692357.02 & 16.24 & -0.01 & -0.88 & -0.87 & 0.23 & -5.32 & 4.34 & $\mathrm{~B} 2 \mathrm{~V}$ \\
\hline $4-15$ & & 53949.38 & -692452.98 & 16.10 & -0.01 & -0.92 & -0.91 & 0.23 & -5.46 & 4.34 & $\mathrm{~B} 2 \mathrm{~V}$ \\
\hline $4-16$ & & 53950.17 & -692313.01 & 13.96 & 0.05 & -0.89 & -0.93 & 0.24 & -7.15 & 4.32 & B1 I \\
\hline $4-17$ & & 53950.72 & -692348.99 & 15.96 & -0.07 & -1.00 & -0.95 & 0.26 & -6.98 & 4.58 & - \\
\hline $4-18$ & & 53950.73 & -692425.98 & 13.66 & -0.05 & -0.98 & -0.94 & 0.26 & -9.57 & 4.61 & O5 If \\
\hline $4-19$ & & 53951.67 & -692412.99 & 16.03 & -0.06 & -0.73 & -0.69 & 0.20 & -5.79 & 4.41 & B1 V \\
\hline $4-20$ & & 53952.26 & -692313.01 & 15.11 & -0.08 & -0.90 & -0.84 & 0.18 & -6.38 & 4.38 & B1 III \\
\hline $4-21$ & & 53952.79 & -69254.00 & 15.83 & -0.11 & -0.91 & -0.83 & 0.15 & -5.76 & 4.41 & B1 V \\
\hline $4-22$ & & 53953.36 & -69251.00 & 15.76 & -0.07 & -0.96 & -0.91 & 0.25 & -6.91 & 4.54 & - \\
\hline $4-23$ & & 53953.77 & -692323.99 & 15.76 & 0.01 & -0.88 & -0.89 & 0.27 & -6.28 & 4.41 & B1 V \\
\hline $4-24$ & & 53954.32 & -692434.01 & 15.66 & -0.10 & -0.98 & -0.91 & 0.14 & -5.97 & 4.41 & B2 III \\
\hline $4-25$ & & 53954.69 & -692456.99 & 15.52 & -0.19 & -0.96 & -0.82 & 0.10 & -6.23 & 4.46 & - \\
\hline $4-26$ & & 53954.89 & -69248.98 & 14.05 & -0.12 & -1.04 & -0.95 & 0.20 & -8.92 & 4.61 & O6.5 V $((f))$ \\
\hline $4-27$ & & 53955.26 & -692437.99 & 15.35 & -0.09 & -0.93 & -0.87 & 0.15 & -5.63 & 4.31 & B2 III \\
\hline $4-28$ & & 53955.46 & -692427.99 & 15.77 & -0.14 & -1.04 & -0.94 & 0.14 & -6.00 & 4.44 & B0.5 V \\
\hline $4-29$ & & 53956.41 & -69242.99 & 15.79 & -0.17 & -0.98 & -0.86 & 0.09 & -5.61 & 4.41 & B1 V \\
\hline $4-30$ & & 53957.16 & -692459.99 & 15.39 & -0.16 & -0.94 & -0.82 & 0.10 & -5.85 & 4.38 & B1 III \\
\hline $4-31$ & & 53957.55 & -692335.01 & 17.30 & 0.03 & -0.71 & -0.73 & 0.27 & -4.39 & 4.34 & $\mathrm{~B} 2 \mathrm{~V}$ \\
\hline $4-32$ & & 53958.12 & -692414.01 & 15.83 & -0.01 & -0.92 & -0.91 & 0.31 & -7.05 & 4.54 & - \\
\hline $4-33$ & & 53958.30 & -692412.99 & 14.42 & -0.08 & -0.94 & -0.88 & 0.24 & -8.59 & 4.60 & $\mathrm{O} 7 \mathrm{~V}$ \\
\hline $4-34$ & & 53958.49 & -692417.00 & 16.54 & 0.02 & -0.98 & -0.99 & 0.30 & -5.73 & 4.44 & B0.5 V \\
\hline $4-35$ & & 53959.63 & -692439.00 & 14.76 & -0.12 & -0.98 & -0.89 & 0.20 & -7.98 & 4.58 & $\mathrm{O} 8 \mathrm{~V}$ \\
\hline $4-37$ & & 53959.63 & -692355.00 & 16.95 & -0.04 & -0.91 & -0.88 & 0.20 & -4.52 & 4.34 & B2 V \\
\hline $4-36$ & & 53959.64 & -692331.99 & 16.56 & -0.08 & -0.90 & -0.84 & 0.16 & -4.46 & 4.31 & B2 III \\
\hline $4-38$ & & 5400.57 & -692447.98 & 16.74 & -0.09 & -0.95 & -0.89 & 0.17 & -4.99 & 4.41 & B1 V \\
\hline $4-39$ & & $\begin{array}{lll}5402.48\end{array}$ & -692338.01 & 15.50 & -0.10 & -1.02 & -0.95 & 0.20 & -6.78 & 4.49 & B0 V \\
\hline $4-40$ & & $\begin{array}{lll}540 & 0.00\end{array}$ & -692439.00 & 15.19 & -0.08 & -0.92 & -0.84 & 0.24 & -7.67 & 4.58 & $\mathrm{O} 8 \mathrm{~V}$ \\
\hline $4-41$ & & 5403.05 & -692410.00 & 13.99 & -0.06 & -0.99 & -0.95 & 0.24 & -8.41 & 4.49 & B0 V \\
\hline $4-42$ & & 5403.24 & -692330.01 & 15.81 & -0.08 & -0.93 & -0.87 & 0.23 & -6.59 & 4.50 & - \\
\hline $4-43$ & & 5403.81 & -692336.00 & 15.71 & -0.08 & -0.87 & -0.81 & 0.16 & -5.64 & 4.34 & B2 V \\
\hline $4-44$ & & 5404.37 & -692414.01 & 15.71 & -0.09 & -0.77 & -0.71 & 0.15 & -5.61 & 4.34 & B2 V \\
\hline $4-45$ & & 5404.94 & -69240.99 & 14.67 & 0.19 & -0.94 & -1.08 & 0.47 & -8.13 & 4.44 & B0.5 V \\
\hline $4-46$ & $14^{w}$ & 5405.89 & -692343.99 & 14.93 & 0.08 & -0.11 & -0.17 & 0.15 & * & * & A3 V \\
\hline $4-47$ & & 5406.46 & -692331.99 & 13.44 & -0.06 & -0.98 & -0.94 & 0.13 & -7.33 & 4.32 & B1 I \\
\hline $4-48$ & $17^{w}$ & 5407.59 & -692420.00 & 15.59 & 1.52 & $*$ & $*$ & 0.58 & * & * & G8 III: ${ }^{f ?}$ \\
\hline $4-50$ & & 5407.97 & -692410.99 & 15.52 & -0.14 & -1.05 & -0.95 & 0.14 & -6.25 & 4.44 & B0.5 V \\
\hline $4-49$ & & 5407.97 & -692338.00 & 17.69 & 0.01 & -0.82 & -0.83 & 0.25 & -3.93 & 4.34 & B2 V \\
\hline $4-51$ & & 5408.92 & -692429.01 & 16.21 & -0.13 & -0.99 & -0.90 & 0.11 & -4.65 & 4.31 & B2 III \\
\hline $4-52$ & & 5409.11 & -69243.98 & 15.33 & -0.10 & -0.97 & -0.90 & 0.22 & -7.18 & 4.53 & - \\
\hline $4-53$ & & $\begin{array}{lll}5 & 40 & 9.49\end{array}$ & -692345.01 & 17.70 & -0.02 & -0.77 & -0.76 & 0.25 & -4.17 & 4.40 & B2: \\
\hline $4-54$ & & 5409.67 & -692422.99 & 13.12 & -0.14 & -1.05 & -0.95 & 0.15 & -9.15 & 4.53 & $\mathrm{O} 8 \mathrm{I}((\mathrm{f}))$ \\
\hline $4-55$ & & 54011.19 & -692311.01 & 14.20 & -0.01 & -0.90 & -0.89 & 0.29 & -8.36 & 4.49 & B0 V \\
\hline
\end{tabular}


Table 2. continued

\begin{tabular}{|c|c|c|c|c|c|c|c|c|c|c|c|}
\hline Id & Other & $\alpha(2000)$ & $\delta(2000)$ & $V$ & $B-V$ & $U-B$ & $Q$ & $E(B-V)$ & $M_{\mathrm{bol}}$ & $\log \left(T_{\text {eff }}\right)$ & Type \\
\hline $4-56$ & $20^{w}$ & 54011.38 & -692357.01 & 13.51 & 0.79 & 0.29 & -0.28 & $*$ & * & * & G3: ${ }^{f}$ \\
\hline $4-57$ & & 54011.38 & -692311.01 & 15.25 & -0.13 & -0.88 & -0.79 & 0.15 & -6.47 & 4.43 & - \\
\hline $4-58$ & & 54011.76 & -692439.01 & 16.91 & 0.96 & * & * & * & * & * & $\mathrm{G} 8-\mathrm{K} 2^{f}$ \\
\hline $4-59$ & & 54012.33 & -692358.00 & 16.10 & 0.05 & -0.94 & -0.98 & 0.29 & -5.65 & 4.34 & B2 V \\
\hline $4-60$ & & 54013.08 & -692415.99 & 16.50 & 0.60 & -0.16 & -0.59 & $*$ & $*$ & $*$ & $\mathrm{~F} 8 \mathrm{~V}$ \\
\hline $4-61$ & $25^{w}$ & 54013.84 & -692318.99 & 13.31 & -0.06 & -0.95 & -0.91 & 0.13 & -7.46 & 4.31 & B1 I \\
\hline $4-62$ & & 54013.65 & -69243.98 & 15.28 & 0.28 & -0.96 & -1.16 & 0.68 & -10.2 & 4.80 & \\
\hline $4-63$ & & 54013.84 & -692422.99 & 15.42 & -0.11 & -0.99 & -0.91 & 0.17 & -6.45 & 4.44 & B0.5 V \\
\hline $4-64$ & & 54013.84 & -69240.99 & 12.24 & 0.54 & 0.26 & -0.13 & $*$ & $*$ & * & $\mathrm{G}^{f}$ \\
\hline $4-65$ & & 54014.79 & -69242.99 & 15.60 & -0.06 & -0.92 & -0.88 & 0.25 & -6.89 & 4.51 & - \\
\hline $4-66$ & & 54015.55 & -692343.99 & 16.15 & -0.05 & -0.89 & -0.85 & 0.19 & -4.96 & 4.31 & B2 III \\
\hline $4-67$ & & 54015.55 & -692410.00 & 15.31 & -0.14 & -0.89 & -0.79 & 0.02 & -4.83 & 4.27 & B2 I \\
\hline $4-68$ & & 54016.49 & -69243.98 & 15.95 & -0.09 & -0.93 & -0.87 & 0.22 & -6.38 & 4.50 & - \\
\hline $4-69$ & & 54017.25 & -692434.01 & 15.99 & 1.27 & 1.05 & 0.14 & * & * & $*$ & G5: ${ }^{f ?}$ \\
\hline $4-70$ & & 53954.49 & -692516.00 & 15.61 & -0.14 & -0.93 & -0.83 & 0.12 & -5.69 & 4.38 & B1 III \\
\hline $4-71$ & & 53945.58 & -692518.01 & 15.10 & -0.13 & -1.03 & -0.94 & 0.13 & -6.50 & 4.41 & B1 V \\
\hline $4-72$ & $11^{w}$ & 5403.04 & -692249.00 & 13.36 & 2.00 & 0.26 & * & $*$ & -7.95 & 3.56 & M I: ${ }^{r v}$ \\
\hline $4-73$ & Sk-69 259 & 54013.65 & -692249.01 & 11.97 & 0.34 & -0.74 & -0.98 & 0.38 & -9.98 & 4.41 & {$[\mathrm{Be}] \mathrm{B} 0^{Z i c}$} \\
\hline $4-74^{*}$ & $12^{l}$ & 53954.90 & -692229 & 13.55 & -0.08 & -0.96 & -0.91 & 0.18 & -9.43 & 4.62 & O4 If \\
\hline $4-100$ & Brey 94 & 53956.24 & -692424.30 & 13.16 & -0.14 & -0.75 & -0.65 & 0.09 & $-10.19^{w r}$ & 4.88 & WC5+O7 \\
\hline $4-101$ & Brey 95 & 5407.75 & -692431.10 & 13.18 & -0.14 & -0.94 & -0.84 & 0.16 & $-10.17^{w r}$ & 4.70 & $\mathrm{WN} 3+\mathrm{O} 7$ \\
\hline $4-102$ & Brey 95a & 54013.50 & -69242.91 & 13.05 & -0.12 & -1.01 & -0.92 & 0.20 & $-10.40^{w r}$ & 4.88 & WC5+O6 \\
\hline
\end{tabular}

Notes to Table 2:

Column 1: Stars 4-70 and 4-71 with pixel coordinates of 202, 165 and 271, 162 in Fig. 5 of Schild \& Testor (1992), 4-72, 4-73 and 4-74 refer to new spectroscopic observations during December 1995 with the B\&C.

In this figure the number 4-68 must be removed and 4-69 and 4-70 replaced by 4-68 and 4-69 respectively.

* This star just outside Zone 4 indicated by an arrow in Fig. 1 presents the same characteristics as $5-31$ : presence of the CIII-CIV $\lambda \lambda 4652-58$ emission lines.

Columns 5, 6 and 7: ${ }^{c} V, B-V$ and $U-B$ values published in Schild \& Testor (1992) were slightly corrected using new photoelectric observations of star 47 and 61, secured by G.T. with the 1-m telescope at ESO the 17 of January 1991.

Column 10: ${ }^{w r}$ integrated $M_{\mathrm{bol}}$ and WR parameters ( $T_{\mathrm{eff}}$ and B.C.).

Column 12: ${ }^{f}$ foreground star, ${ }^{f}$ ? according to the anomalous colors the star should be foreground while its spectrum shows that it should be member of LH 104. Better spectral resolution spectra are needed for such a star that could be a late supergiant or a combination of an unreddened $\mathrm{O}$ star and a late star. ${ }^{r v} \mathrm{~A} \mathrm{RV}_{\text {hel }}$ of $262.6 \mathrm{~km} / \mathrm{s} \pm 1.9$ was obtained with CORAVEL (Lortet et al., in preparation).

Zic Spectral type given by Zickgraf et al. (1986). Due to emission lines the $V$ and $B-V$ used for the $M_{\text {bol }}$ computation are 12.22 and 0.14 respectively, and give a lower limit of $M_{\mathrm{bol}}$.

emphasizes the low-ionisation structure and shows a long and weak filament surrounding the OB association. The $\mathrm{H} \beta$ image of $\mathrm{N} 158 \mathrm{C}$ shows the HII region composed of the three bright excited zones 1, 2 and 3 which are best seen in the [OIII] image (Fig. 3).

\section{Spectral classification}

The spectral types of the numerous OB stars present in both LH 104 and LH 101 were determined comparing our data with Walborn \& Fitzpatrick's (1990) atlas of digital spectra. Further details of the spectral classification are described in Paper I. The galactic stars were discriminated by their approximate heliocentric near zero radial velocity. In Fig. 4 the spectra of a few evolved and unevolved OB stars are plotted in the range of $\lambda \lambda 4000$ to $4800 \AA$.

\subsection{Individual interesting stars}

\subsubsection{The O3If star 5-31(=Westerlund 19)}

The star 5-31 (W19) coincides with the location of the NIR 1 source (Jones et al. 1986). Its optical spectrum shows a narrow NIV $\lambda 4058 \AA$ emission feature and NV $\lambda \lambda 4604,4620 \AA$ absorption lines (Fig. 4). The NIV $\lambda 4058$ emission line is slightly stronger than NIII $\lambda 4640$ emission, 


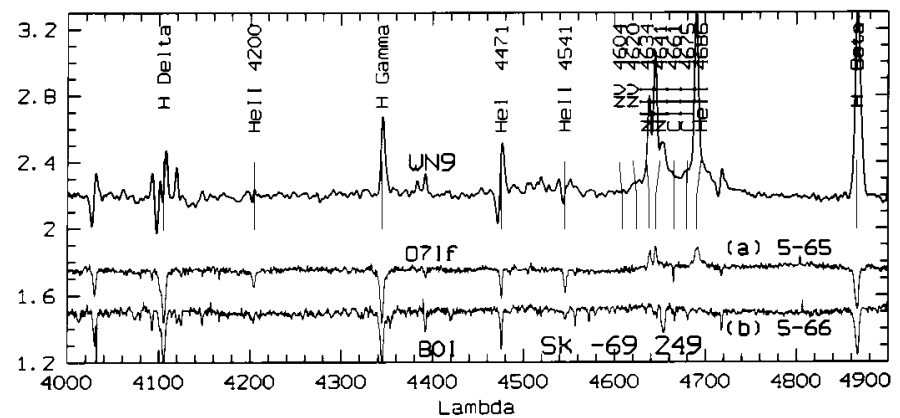

Fig. 3.1.1a. Selected rectified spectra of the components a) and b) of the complex star Sk -69 249. b) and the WR Brey 91

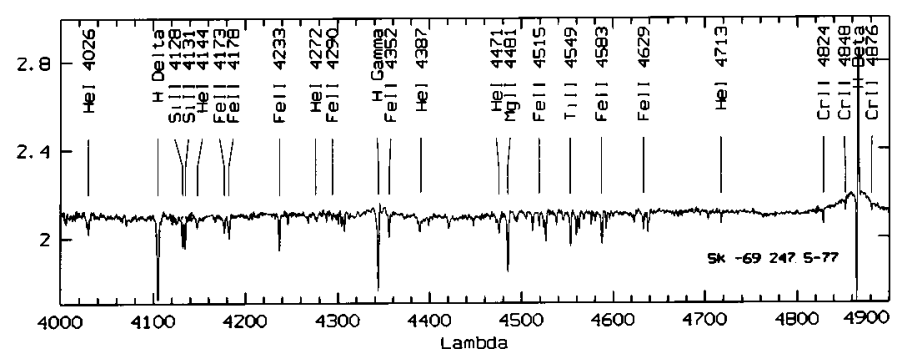

Fig. 5b. Rectified spectrum of Sk -69 247

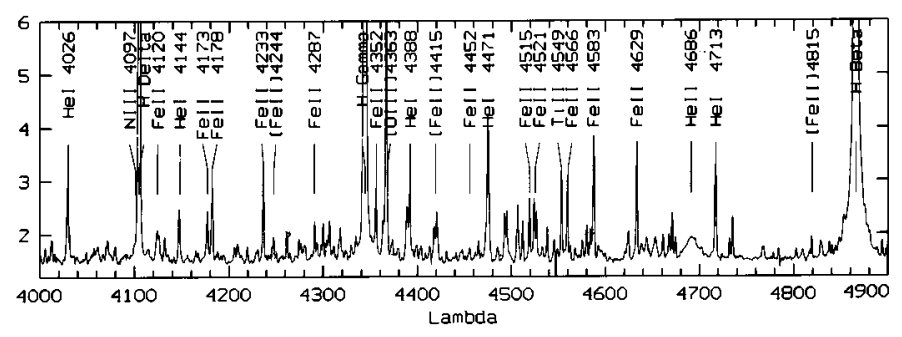

Fig. 5c. Rectified spectrum of the [Be] star Sk -69259 showing sharp emission lines. Note also the broader He II $\lambda 4686$ emission. A few emission lines of FeII and [FeII] are identified

and with the HeII $\lambda 4686 \AA$ line also in emission this star presents the characteristics of an O3 supergiant which we classify O3 If*. It is also interesting to note the presence of CIII-IV $\lambda 4652$ emission lines similar to NIII $\lambda 4634-41$.

\subsubsection{Stars $5-65$ and $5-66=\mathrm{Sk}-69249$}

This star, located $3^{\prime \prime}$ east of the Wolf-Rayet star Brey 91 of type WN9 (Breysacher 1981), appears to consist of two components (a) and (b) of similar magnitude separated by only $1.6^{\prime \prime}$ (Fig. 3). The spectrum of the northern component $(a)=5-65$ shows HeII $\lambda 4686$ and NIII $\lambda 4640$ in emission (Fig. 3.1.1a). The HeI $\lambda 4471$ appears similar to HeII $\lambda 4541$, thus we classify this star as O7 If. From several spectra obtained during consecutive nights, the emission lines of NIII and HeII show large amplitude radial velocity variations. This star probably is a spectroscopic binary. The spectrum of the southern compo-

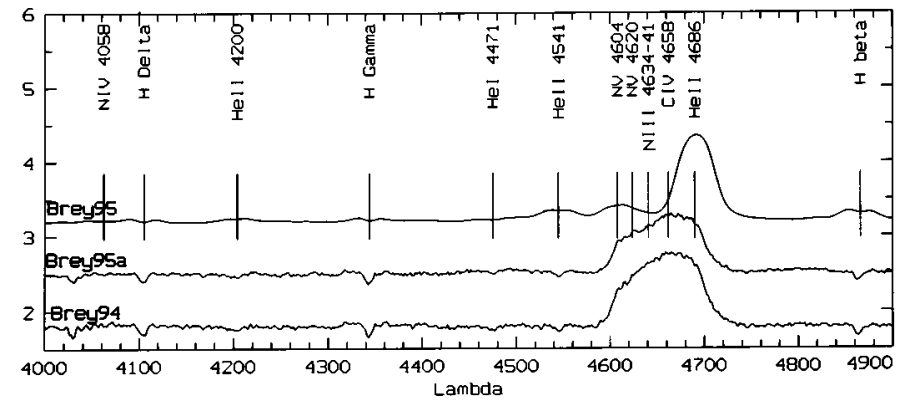

Fig. 5d. Spectrograms of the three WR star systems in LH104

nent $(b)=5-66$ shows rather strong absorptions of SiIV $\lambda \lambda 4089,4116$, SiIII $\lambda \lambda 4552,68,75$ and the blend OII and CIII $\lambda 4650$, thus we classify it as a supergiant B0 I. Without sufficient spatial resolution, this star contaminates the northern component.

\subsubsection{Star $5-76=$ Sk $-69247=$ HD 269923}

The visually brightest star in LH 101, HD 269923 has been

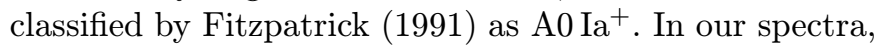
P Cygni profiles are well seen in $\mathrm{H} \beta$ and $\mathrm{H} \gamma$. Particularly prominent is the $\mathrm{P}$ Cyg profile in $\mathrm{H} \beta$, with extended emission wings (Fig. 3.1.1b). This kind of profiles are often observed in Luminous Blue Variable (LBV) type stars (e.g. Walborn 1997). The spectrum of HD 269923 also presents many strong photospheric absorption lines of Fe II.

\subsubsection{Star $4-73=$ Sk $-69259=$ HD $38489=$ S 134}

This luminous star located at the northern edge of the association LH 104, was studied by Shore \& Sanduleak (1983). Our spectrum of $\delta \lambda \sim 1 \AA$ (Fig. 5c) shows very narrow emissions of low-excitation metals, and strong nebular and Balmer emission lines. As the variations of these strong lines affect the photometry the $V$ and $B-V$ magnitudes have to be corrected by 0.24 and 0.20 , respectively (Zickgraf et al. 1986). The widths of a few lines are listed in Table 3. In order to have a good accuracy on sharp lines the third column indicates $F W H M$ values obtained after deconvolution by a gaussian of $F W H M=$ $1 \AA$. These characteristics with an $\mathrm{H} \beta$ equivalent width of $\sim 200 \AA$ confirm the $\mathrm{B}[\mathrm{e}]$ supergiant type of this star described by Zickgraf (1990). Our spectrum has rather better signal/noise ratio than those published previously, and allows to point out very well the presence of a relatively weak and broad HeII $\lambda 4686$ emission line of $F W H M=$ $15.2 \AA$, an indication for a hot, high velocity stellar wind (Zickgraf et al. 1986). 
Table 3. Emission line widths in the spectrum of HD 38489

\begin{tabular}{lll}
\hline Line & FWHM $(\AA)$ \\
& & \\
\hline $\mathrm{H} \beta$ & 2.00 & 1.70 \\
HeII $\lambda 4686$ & 15.2 & 15.16 \\
H $\lambda 4101$ & 1.95 & 1.67 \\
He I $\lambda 4713$ & 1.82 & 1.52 \\
[OIII] $\lambda 4363$ & 1.52 & 1.14 \\
{$[$ Fe II] $\lambda 4287$} & 1.11 & 0.48 \\
Fe II $\lambda 4233$ & 1.38 & 0.95 \\
\hline
\end{tabular}

\section{Age structure of LH 101/104}

\subsection{Transformation to $M_{\mathrm{bol}}, T_{\mathrm{eff}}$ for known spectral type stars}

The stellar energy distribution derived by color calibration is not accurate due to uncertainties of the bolometric magnitude $\left(M_{\text {bol }}\right)$ for hot stars with $\log T_{\text {eff }}>4.4$ (Massey et al. 1995). Therefore we need to place these stars in the $\mathrm{H}-\mathrm{R}$ diagram with the help of spectral types obtained by spectroscopy.

We deduced values of $E(B-V), M_{\mathrm{bol}}$ and $\log T_{\mathrm{eff}}$ which are listed in Tables 1 and 2 where Cols. 1 and 2 give the stellar identification, and Col. 3 the epoch 2000 coordinates. We adopted a distance modulus $m-M=18.5$ (e.g. Panagia et al. 1991) for the LMC, and an ordinary ratio $R=3.1=A_{V} / E(B-V)$.

The $(B-V)_{0}$ intrinsic color indices, the bolometric corrections $(\mathrm{BC})$ and $T_{\text {eff }}$ for different spectral types were taken from Schmidt-Kaler (1982), except for O stars for which the values from Chlebowski \& Garmany (1991) were used. For WR stars the $T_{\text {eff }}$ is from Hamann et al. (1991) and Koesterke et al. (1991) and BC from Smith \& Maeder (1989).

\subsection{Transformation to $M_{\mathrm{bol}}, T_{\mathrm{eff}}$ for unknown spectral type stars}

For stars of unknown spectral type, we first determined the color factor $Q=(U-B)-0.72(B-V)$, a coarse indicator of spectral type and then derived the stellar parameters following the procedure of Massey et al. (1995).

The differences between the observational and the theoretical values of the $Q$ parameter listed in Col. 7 of Tables 1 and 2 , show that in a nebula and bad photometric conditions this parameter is not reliable for hot stars, contrary to the spectroscopy classification (e.g. $Q \sim-0.80$ for the stars 5-31 and 5-52 instead of $\sim-0.95)$.

\subsection{Hertzprung-Russel diagrams of LH 101 and LH 104}

The resulting $\mathrm{H}-\mathrm{R}$ diagrams $\log T_{\text {eff }}$ vs. $M_{\text {bol }}$ for both $\mathrm{OB}$ associations are shown in Figs. 4.1a and b. We dis-
Table 4. Distribution of stars with isochrones

\begin{tabular}{llllll}
\hline$M_{\odot}$ & \multicolumn{5}{l}{ Myr } \\
& $0-2$ & $2-4$ & $4-6$ & $6-8$ & $8-10$ \\
\hline $85-120$ & 1 & 2 & & & \\
$60-85$ & 1 & & & & \\
$40-60$ & 3 & 2 & 2 & & \\
$25-40$ & 1 & 2 & 3 & 2 & \\
$15-25$ & & & 4 & 7 & 6 \\
\hline & & & LH 104 & & \\
$85-120$ & & & & & \\
$60-85$ & & 1 & & & \\
$40-60$ & & 7 & 1 & & \\
$25-40$ & & 4 & 2 & 1 & \\
$15-25$ & 1 & 3 & 4 & 1 & 2 \\
\hline
\end{tabular}

Notes to Table 4:

Column 1: Masses

Columns 2, 3, 4, 5, 6: Number of stars per 2 Myr interval in each mass bin.

criminated between stars with spectral classifications and those with photometry only, denoting them with different symbols. We also overlaid a few stellar evolutionary tracks and isochrones calculated by Schaerer et al. (1993) for a metallicity of $Z=0.008$.

In Tables 1 and 2 the apparent magnitudes and the colors of the three WR + O systems refer to the observed integrated magnitude, as is the case for the spectroscopic binary star 5-65 of type O7If. The composite spectra are shown in Fig. 5 a and Fig. 5 d. The $M_{\text {bol }}$ derived from these integrated magnitudes does not have any clear meaning. However these stars should be taken into account in the HR diagram and the IMF. As a first approximation, since spectra of both components are visible, we have assumed that these systems are composed of two stars of similar magnitudes, and that the $\mathrm{O}$ components in the WR systems are of luminosity class $V$.

In the HR diagrams of Figs. 4.1a and b, we have plotted the positions of the $\mathrm{O}+\mathrm{O}$ and $\mathrm{WR}+\mathrm{O}$ systems as they would appear with the $M_{\text {bol }}$ values which result from the apparent integrated magnitude and $T_{\text {eff }}$ of the $\mathrm{O}$ type component. This illustrates how the stars would appear in the HR diagram if we had no information about their binarity.

Arrows are drawn from these positions in the HR diagram to where the components would be located assuming two equal magnitude stars of the observed spectral types. In LH 104 this procedure locates the $\mathrm{WR}+\mathrm{O}$ systems in the bin $40-60 M_{\odot}$.

\subsection{Age}

LH 101 seems to consist of two subpopulations (Table 4). A comparison with the isochrones shows that the younger 


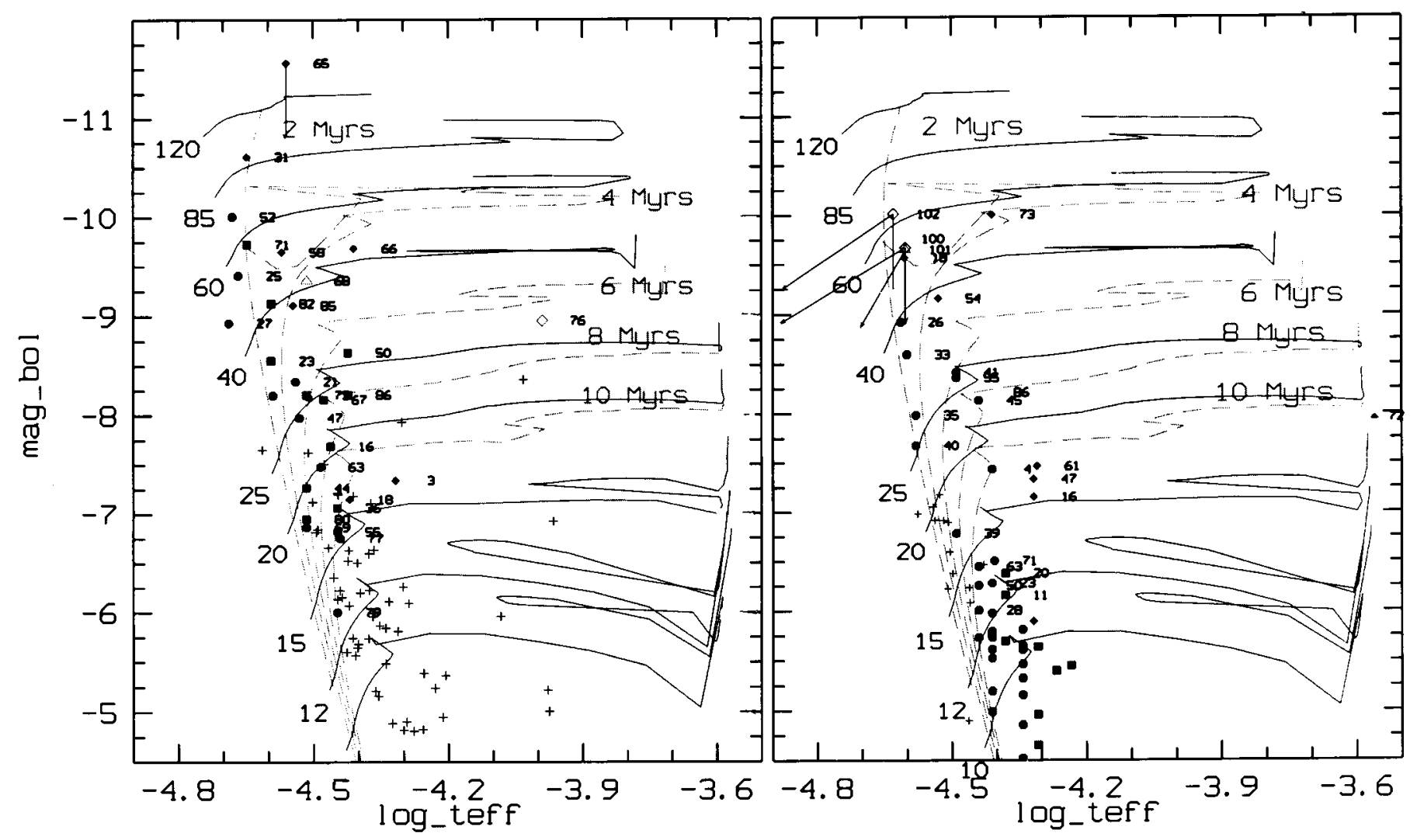

Fig. 6. a) and b) are the HR diagrams of the OB associations LH101 and LH104. Filled circles, squares and diamonds show data with spectral classification and they indicate luminosity $V$, III and I respectively. The crosses show data determined only by photometry. Empty diamonds indicate WC types and empty triangles WN types. For O star belonging to systems the $M_{\text {bol }}$ obtained from observed integrated magnitude is represented by filled triangles. The stellar evolutionnary tracks with the corresponding stellar mass identified in $M_{\odot}$ and isochrones in dashed lines are overlaid (Shaerer et al. 1993)

subgroup has an age of $\leq 2 \mathrm{Myr}$ and the older one of $\sim 3-$ 6 Myr. As noted by Testor \& Niemela (1996), stars outside the nebulosity seem to be somewhat more evolved than those inside the nebulosity. Thus sequential star formation appears to be present even at scales less than $50 \mathrm{pc}$.

LH 104 consists mainly of a young population spreading out between 2 and 6 Myr (Table 4). The HR diagrams also show in both associations a group of older and less massive stars.

\section{IMF}

We used the term initial mass function (IMF) for an observed mass function that is already evolved and we assume that the stars are coeval (Parker \& Garmany 1993) although if we refer to our previous and present results (Testor \& Schild 1993) this is not completely valid. To construct the IMF we counted between mass tracks shown in the H-R diagram the number of stars. Using Scalo's (1986) notation, the slope of the initial mass function is $\Gamma=\mathrm{d} \log \xi(\log m) / \mathrm{d} \log m$

where $m$ is the stellar mass in units of the solar mass and the mass function $\xi=\xi\left[(\log m)^{-1} \mathrm{kpc}^{-2}\right]$ is the number of stars born per square kiloparsec and per unit logarithmic mass interval.

We combine stars with known spectral types and stars of which $M_{\text {bol }}$ and $T_{\text {eff }}$ were derived by photometry only and consider that the incompleteness becomes significant below $10 M_{\odot}$. We find IMF slopes of $\Gamma=-1.29 \pm 0.20$ and $\Gamma=-1.05 \pm 0.12$ for LH 101 and LH 104, respectively. The IMF slope for LH101 is close to that of Salpeter (1955). As its stars constitute two age groups the IMF should represent rather the PDMF (Massey 1997), i.e. the mass function of the massive stars observed at the present day. The derived mass function $\xi$ is shown in Figs. 5a and b. The flatter slope of the IMF in LH 104 is probably a consequence of our approximate method in deriving the parameters of the $\mathrm{O}$ and WR components in the massive binary systems. Some of these systems could contain more than two stars.

The IMF slopes found are comparable with those of numerous LMC OB associations in the LMC studied by different authors and listed by Massey (1997). 

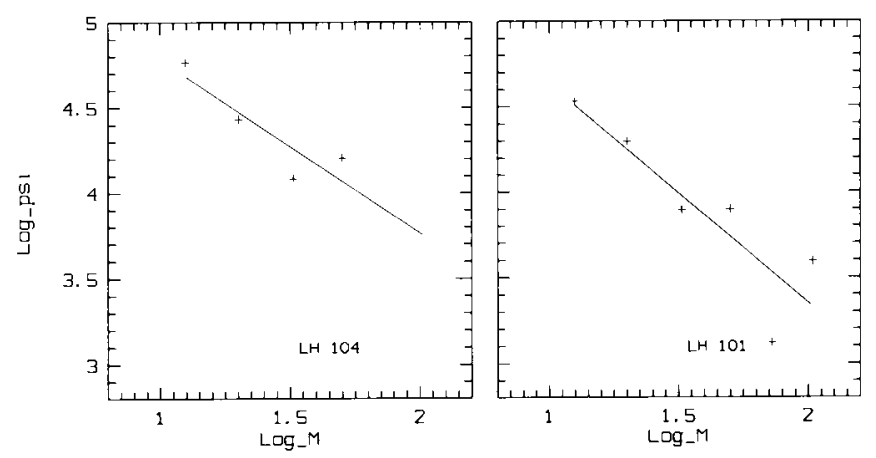

Fig. 7. a-b) The initial mass function for LH 104 and LH 101. The slope determined from a fit to the mass bins with $10 M_{\odot} \leq M \leq 60 M_{\odot}$ is represented by a dashed line and with $10 M_{\odot} \leq M \leq 85 M_{\odot}$ by a solid curve

\section{Ho flux in LH 101}

Absolute $\mathrm{H} \alpha$ photometry observations (Caplan \& Deharveng 1985) in an aperture of $4.9^{\prime}$ give an $\mathrm{H} \alpha$ flux of LH 101 equivalent to a number of Lyman continuum photons per second $\left(N_{\mathrm{Lyc}}^{\mathrm{obs}}\right)$ of $11210^{48}$ photons/s (Devereux \& Scowen 1994) and corresponding to $\sim 3$ O V stars (Panagia 1973). The number of Lyman continuum photons derived from our quasi complete $\mathrm{O}$ star content of LH $101\left(N_{\mathrm{Lyc}}^{\mathrm{P}}\right)$ (Table 5) in the same area (Fig. 3) is found to be larger $\left(N_{\mathrm{Lyc}}^{\mathrm{P}}=42010^{48} \mathrm{ph} / \mathrm{s}\right)$ corresponding to $\sim 10 \mathrm{OV}$.

As seen in Fig. 3, the HII region is composed of three distinct zones. Using a few IDS nebular spectra of bright regions of LH 101 obtained with the ESO 1.5-m telescope in 1984, and our monochromatic CCD $\mathrm{H} \beta$ image (Fig. 3), we calibrated each zone in flux $F(\mathrm{H} \beta)$ (Table 5).

We notice that as well in each HII zone as in the whole HII region, the $N_{\mathrm{Lyc}}^{\mathrm{P}}$ derived from the $\mathrm{O}$ stars is greater than $N_{\text {Lyc }}^{\text {obs }}$ derived from the observed $\mathrm{H} \alpha$ or $\mathrm{H} \beta$ flux by a factor 3-4. This discrepancy could be both attributed to inhomogeneity in the gas that would let escape Lyman continuum photons and to dust obscuration sensitive to $\mathrm{H} \alpha$ (Caldwell \& Kutner 1996). The reality of this density inhomogeneity is a basic property of young HII regions. Here we notice that the stellar population is strongly different in the $\mathrm{S}-\mathrm{E}$ active $\mathrm{HII}$ region populated by unevolved $\mathrm{O}$ stars, and in the N-W and towards LH 104 where stellar winds probably have dispersed the interstellar matter.

\section{Summary}

We combined new observations of the LH 101 OB association with the previously published data of LH 104, in order to construct upper $\mathrm{H}-\mathrm{R}$ diagrams of the stellar population of the two regions. We find the following:

- LH 101 consists of at least two age groups, one with an age of $\leq 2 \mathrm{Myr}$ mainly composed of stars inside the nebulosity, and the other of stars outside the nebulosity
Table 5. Number of Lyman continuum photons $N_{\mathrm{Lyc}}^{\mathrm{P}}$ derived from different Zones

\begin{tabular}{lllllll}
\hline & & & & & & \\
$\mathrm{N}^{\circ}$ & $F(\mathrm{H} \beta)$ & $N_{\text {Lyc }}^{\text {obs }}$ & Ident & Type & $N_{\text {Lyc }}^{\mathrm{P}}$ & $C(\mathrm{H} \beta)$ \\
& & & & & & \\
\hline 1 & 0.33 & 2.31 & W19 & O3If & 8.5 & 0.43 \\
2 & 0.51 & 3.57 & W20 & O4 & 12.5 & 0.50 \\
& & & W24 & O5 V((f)) & & \\
3 & 0.35 & 2.45 & W7 & O4-5III(f) & & 0.47 \\
& & & W9 & O9.5III & 9.5 & \\
\hline
\end{tabular}

Notes to Table 5:

Column 1, 2: Zone numbering, $F(\mathrm{H} \beta)$ in $10^{-10} \mathrm{erg} \mathrm{s}^{-1} \mathrm{~cm}^{-2}$ in an aperture of $30^{\prime \prime}$.

Column 3: $N_{\mathrm{Lyc}}^{\text {obs }}$ in $10^{49}$ photons $\mathrm{s}^{-1}$ corrected for the interstellar extinction.

Column 4: Westerlund identification.

Column 5: Spectral type.

Column 6: $N_{\mathrm{Lyc}}^{\mathrm{P}}$ derived from the ionizing stars (Panagia 1973).

Column 7: Extinction $C(\mathrm{H} \beta)$ from $\mathrm{H} \alpha / \mathrm{H} \beta$.

with an age of 3-6 Myr. LH 104 appears to be composed only of one group of $2-6 \mathrm{Myr}$.

- The IMF slope of LH 101 with $\Gamma \leq-1.29 \pm 0.20$ seems to be well represented by a Salpeter slope, whereas for LH 104 the IMF slope is flatter with $\Gamma \sim-1.05 \pm 0.12$, which probably is the result of the presence of the binary systems containing WR stars.

- LH 101 contains within the nebulosity to the S-E several reddened unevolved early $\mathrm{O}$ type stars, and in the $\mathrm{N}-\mathrm{W}$ portion unreddened evolved massive stars outside the nebulosity, suggesting that even in LH 101 a propagating star formation is present, probably on a scale of the order of $50 \mathrm{pc}$.

- The ionizing flux provided by the early $\mathrm{O}$ stars present in $\mathrm{N} 158 \mathrm{C}$ is in excess of that derived from the $\mathrm{H} \beta$ luminosity of our narrow band image. Therefore, the $\mathrm{H} \beta$ or $\mathrm{H} \alpha$ luminosity may not be a reliable indicator of stellar content in sites of massive star formation.

\section{References}

Breysacher J., 1981, A\&A 149, 213

Caldwell D.A., Kutner M.L., 1996, ApJ 472, 611

Caplan J., Deharveng L., 1985, A\&AS 62, 63

Chlebowski T., Garmany C.D., 1991, ApJ 368, 241

Devereux N.A., Scowen P.A., 1994, AJ 108, 1244

Fitzpatrick E., 1991, PASP 103, 1123

Hamann W.R., Dunnebeil G., Koesterke L., Schmutz W., Wessolowski U., 1991, A\&A 249, 443

Henize K.G., 1956, ApJS 2, 315

Israel F.B., Maloney P.R., Geis N., Herrmann F., Madden S.C., Poglitsch A., Stacey G.J., 1996, ApJ 465, 738

Jones J.J., Hyland A.R., Straw S., Harvey P.M., Wilking B.A., Joy M., Gatley I., Thomas J.A., 1986, MNRAS 219, 603

Kennicut R.C., Hodge P.W., 1986, ApJ 306, 130 
Koesterke L., Hamann W.R., Schmutz W., Wessolowski U., 1991, A\&A 248, 166

Landolt A.U., 1992, AJ 104, 340

Lortet M.C, Testor G., 1988, A\&A 194, 11

Lortet M.C, Martin N., Maurice E., Testor G., 1997 (in preparation)

Lucke P., 1972, Ph.D. Thesis, Univ. Washington

Lucke P., Hodge P.W., 1970, AJ 75, 171

Massey P., 1997, "The stellar initial mass function" proceedings of the 38th Herstmonceux Conference, Gilmore G., Parry I., and Ryan S. (eds.) (in press)

Massey P., Lang C.C., Degioa-Eastwood K., Garmany C.D., 1995, ApJ 438, 188

Melnick J., Dekker H., D’Odorico S., 1989, ESO operating manual $\mathrm{N}^{\circ} 4$

Panagia N., 1973, AJ 78, 929

Panagia N., Gilmozzi R., Macchetto F., Adorf H.-M., Kirshner R.P., 1991, ApJ 380, L23

Parker J.Wm., Garmany C.D., 1993, AJ 106, 1471

Sanduleak N., 1969, Cerro Tololo Inter-American Obs. Contr. $\mathrm{N}^{\circ} 89$

Salpeter E.E., 1955, ApJ 121, 161

Scalo J.M., 1986, Fundam. Cosmic Phys. 11, 1

Schaerer D., Meynet G., Maeder A., Schaller G., 1993, A\&A 98,523
Schild H., Testor G., 1992, A\&AS 92, 729

Schmidt-Kaler T., 1982, Landolt-Bornstein, New Series, Group VI 2 , p. 14

Shore N.S., Sanduleak N., 1983, AJ 273, 177

Smith L.F., Maeder A., 1989, A\&A 211, 71

Stetson P.B., 1987, PASP 99, 191

Testor G., Niemela V., 1996, in: D. Kunth, B. Guiderdoni, Heydari-Malayeri M. \& Thuan T.X. (eds.), The Interplay between Massive Star Formation, the ISM and Galactic Evolution, Proc. 11th IAP Meeting. Gif-sur-Yvette: Éditions Frontières, p. 487

Testor G., Schild H., 1993, A\&A 280, 426

Walborn N.R., 1997, in: Nota N. (ed.), Luminous Blue Variables: Massive Stars in Transition, ASP Conf. Ser. (in press)

Walborn N., Fitzpatrick E., 1990, PASP 102, 379

Westerlund B., 1961, Uppsala Astron. Obs. Ann. 5, No 1

Zickgraf F.J., 1990 in Angular Momentum and Mass Loss for Hot stars, Willson L.A. \& Stalio R. (eds.). Dordrecht: Kluwer, p. 245, Z90

Zickgraf F.J., Wolf B., Stahl O., Leitherer C., Appenzeller I., 1986, A\&A 163, 119 